\title{
The leukemia associated ETO nuclear repressor gene is regulated by the GATA-1 transcription factor in erythroid/megakaryocytic cells
}

\author{
Ram Ajore1, Rakesh Singh Dhanda*2, Urban Gullberg ${ }^{1}$ and Inge Olsson ${ }^{1}$
}

\begin{abstract}
Background: The Eight-Twenty-One (ETO) nuclear co-repressor gene belongs to the ETO homologue family also containing Myeloid Translocation Gene on chromosome 16 (MTG16) and myeloid translocation Gene-Related protein 1 (MTGR1). By chromosomal translocations ETO and MTG16 become parts of fusion proteins characteristic of morphological variants of acute myeloid leukemia. Normal functions of ETO homologues have as yet not been examined. The goal of this work was to identify structural and functional promoter elements upstream of the coding sequence of the ETO gene in order to explore lineage-specific hematopoietic expression and get hints to function.

Results: A putative proximal ETO promoter was identified within 411 bp upstream of the transcription start site. Strong ETO promoter activity was specifically observed upon transfection of a promoter reporter construct into erythroid/ megakaryocytic cells, which have endogeneous ETO gene activity. An evolutionary conserved region of $228 \mathrm{bp}$ revealed potential cis-elements involved in transcription of ETO. Disruption of the evolutionary conserved GATA -636 consensus binding site repressed transactivation and disruption of the ETS1 -705 consensus binding site enhanced activity of the ETO promoter. The promoter was stimulated by overexpression of GATA-1 into erythroid/megakaryocytic cells. Electrophoretic mobility shift assay with erythroid/megakaryocytic cells showed specific binding of GATA-1 to the GATA -636 site. Furthermore, results from chromatin immunoprecipitation showed GATA-1 binding in vivo to the conserved region of the ETO promoter containing the -636 site. The results suggest that the GATA - 636 site may have a role in activation of the ETO gene activity in cells with erythroid/megakaryocytic potential. Leukemia associated AML1ETO strongly suppressed an ETO promoter reporter in erythroid/megakaryocytic cells.

Conclusions: We demonstrate that the GATA-1 transcription factor binds and transactivates the ETO proximal promoter in an erythroid/megakaryocytic-specific manner. Thus, trans-acting factors that are essential in erythroid/ megakaryocytic differentiation govern ETO expression.
\end{abstract}

\section{Background}

The human ETO co-repressor family comprises the homologous nuclear proteins ETO (Eight-Twenty-One), MTG16 (Myeloid Translocation Gene on chromosome 16) and MTGR1 (Myeloid translocation Gene-Related protein1) evolutionary related to the Drosophila protein Nervy [1]. The ETO homologues do not interact directly with DNA but are recruited by transcription factors such as PLZF, BCL6, TAL1/SCL, Gfiland Heb [2-7] to become partners of multi-protein complexes on a gene promoter

* Correspondence: Rakesh.Singh@med.lu.se

2 Protista Biotechnology AB, IDEON, Ole Römers väg 12, SE 22370 Lund, Sweden

Full list of author information is available at the end of the article
[8,9]. The ETO homologues of the complexes recruit nuclear co-repressors such as N-CoR, [9-11] SIN3 $[9,10,12]$ and SMRT $[8,13]$, which in turn interact with histone deacetylase (HDAC) compelling transcriptional repression.

Importantly, ETO homologue genes are commonly involved in reciprocal chromosomal translocation $(\mathrm{t})$ characteristic of acute leukemia. For example, the ETO gene becomes fused to the $A M L 1$ (Runx1) transcription factor gene by $\mathrm{t}(8 ; 21)$ resulting in the biosynthesis of the AML1-ETO fusion protein [14,15]. Similarly, the MTG16 gene becomes fused to the $A M L 1$ gene by $\mathrm{t}(16 ; 21)$ resulting in the production of the AML1-MTG16 fusion protein [16]. The oncogenic fusion proteins interfere with 
hematopoietic gene regulation by transcriptional repression mediated by ETO and MTG16, respectively. Corepressors-HDAC recruited by the ETO portion of AML1-ETO diminishes chromatin accessibility leading to transcriptional repression at AML1 targets [8-10], contributing to the cellular differentiation block of the leukemic cells.

Gene expression involves both trans-acting factors such as transcription factors and cis-acting elements such as promoter, enhancer and silencer regions whose accessibility to the trans-acting factors is governed by the chromatin packing. The mechanisms for transcriptional on/ off switching of ETO homologue genes have not been examined. ETO and MTG16 show distinct cell-type-specific expression suggesting differences in gene regulation. ETO is present in many normal tissues with the highest transcript level detected in brain and heart $[17,18]$. MTG16 is expressed for example in hematopoietic tissues, placenta and pancreas [18]. Furthermore, the ETO homologues are differently expressed during hematopoietic differentiation; ETO is transiently expressed during erythropoiesis, MTG16 is expressed in progenitor cells and downregulated during myeloid and erythroid differentiation and MTGR1 is ubiquitously expressed, further suggesting differences in gene regulation among the ETO homologues [19]. Results from gene targeting reveals involvement in hematopoietic development of MTG16 [20] but not of ETO [21] or MTGR1 [22]. The leukemogenic fusion protein AML1-ETO promotes self renewal of primary erythroid cells [23] concomitant with an AML-ETO-induced block of erythroid lineage commitment. This block correlates to blockade of p300/CBP coactivation complex-mediated acetylation of the erythroid regulatory transcription factor GATA-1 [24]. As ETO is expressed in human erythroid cells [19] it may be affected by AML1-ETO.

The restriction of hematopoietic expression of ETO to erythroid cells [19] suggests an involvement in lineagespecific gene regulation. In order to study lineage-specificity it is essential to identify structural and functional promoter elements upstream of the coding sequences of the ETO gene. As erythroblasts and megakaryocytes derive from a common bipotent erythroid/megakaryocyte progenitor [25] studies were done in both cell types. Our results show a critical role for an evolutionary conserved GATA binding site in transcriptional regulation of the ETO gene in cells of erythroid/megakaryocytic potential.

\section{Results}

Homologous non-coding ETO sequence in human, mouse and rat

In order to identify the location of the proximal ETO promoter, the transcription start site was identified by use of 5'-rapid amplification of cDNA-ends (RACE) with mRNA extracted from HEL cells. The amplified cDNA was cloned, sequenced and aligned to genomic DNA (Fig. 1A). A complete sequence match with an upstream region of the ETO transcript variant-3 (NCBI Ref. Seq: NM_175635.1) was observed and the transcription start site was identified at -318 bp (translational start codon at +1 ). Orthologous genes may be subject to similar regulatory mechanisms in conserved regions of different species [26]. A search for homologies within the gene upstream of the transcription start revealed a region at 659 to -432 bp that was highly conserved between human, mouse and rat (Fig. 1B). This region may carry important cis-acting regulatory elements. Examination by bioinformatics analysis revealed potential ETS1 binding sites (5'-TTTCCT-3') at -705 and -661; GATA consensus transcription factor-binding sites at -651 (5'CCATCT-3'), -636 (5'-TGATA-3'), and -619 (5'TGATGC-3') and a CAAT binding site at -633 (5'TATTG-3') (Fig. 1B).

\section{Functional promoter upstream of the ETO coding region with erythroid/megakaryocytic specificity}

The factors regulating the ETO gene expression have to our knowledge not been determined. Therefore, we aimed at identifying major regulatory cis- acting regions and trans- acting factors regulating human ETO expression in hematopoietic cells. To examine whether the sequence upstream of the transcription start of the ETO gene is transcriptionally active, we cloned an -1820 to 259 bp region (translational start codon at +1 ), which was inserted upstream of the luciferase reporter gene in promoterless pGL3/Basic vector, creating the plasmid pGL3/ -1820-259. We transfected plasmids into hematopoietic cell lines and determined luciferase activity. Transcriptional activity was normalized to pGL3/SV40-promoter; promoterless pGL3/Basic served as negative control. Renilla vector was used as internal control for transfection efficiency. pGL3/-1820 to -259 showed an approximately 3-fold increased reporter signal compared to pGL3/SV40-promoter both in erythroid (HEL) and megakaryocytic (MEG-01) cell lines (Fig. 2, top) suggesting the presence of strong cis-regulatory elements in this particular region.

In order to identify the functionally important regulatory DNA sequences, sequential deletions were made from the 5 'end of the -1820 to $-259 \mathrm{bp}$ region. The deletions were inserted upstream of the luciferase reporter gene in promoterless pGL3/Basic thus generating pGL3 1326-259, pGL3 -839-259, pGL3 -729-259, pGL3 -579259 , and pGL3 -429-259 reporter constructs, which were transfected into erythroid HEL and megakaryocytic MEG-01 cell lines. The region between -729 and -259 bp was found to retain the transcriptional activity (Fig. 2). 
A

\title{
Transcription start site $\downarrow$
}

GATAAGACCAGGAGAAGTGAAGATGTAACATGTTATCTGTCGCTCCTCTTAGCTGGCGGAGAGAATTTACA - 318 GATAAGACCAGGAGAAGTGAAGATGTAACATGTTATCTGTCGCTCCTCTTAGCTGGCGGAGAGAATTTACA

TTTAAAGATTAGCAGAGTGAGAAAGAGAAATCTGCCTTTTGTTGTGTGGGGTGAGGAGGAGGCATCTACCC -247 TTTAAAGATTAGCAGAGTGAGAAAGAGAAATCTGCCTTTTGTTGTGTGGGGTGAGGAGGAGGCATCTACCC CTGGCCTTGACGCTATCTCCCATCACCTCTGCTATCCAGACAGGACTCACCGAGGTGAGAAT - - - - - - - - 176 CTGGCCTTGACGCTATCTCCCATCACCTCTGCTATCCAGACAGGACTCACCGAGGTGAGAATACCGGAGGG

- 105 CCTTATCTTTAATTGGGTTTAGTTTTGCCAGTCTGAATAGGTTTAAAGAGACTCGATAAAGGGGGAACAAT

- 34 AgAtTAtTtATtgactggacgCtgAagcctTtAgatgaAgA Genome

B

ETS1 - 705

ETS1 - 661 - 729 TGTCTTCTCTCTCTCTCTCCCACTTTTCCTCCCTCTCCCGCTCCGTCTCACACGCACCCTCTGTTTATTTT CAAT - 633

GATA - 651 GATA - 636 GATA - 619

- 658 СCTGCCT

-587 GCGCTCACACACTCACTCACACACAATGTGCCATCCTGACAAGGCTTTTACTTCTGATAAGCTCCAATGTG

- 516 TGTTTAATGAATACAAAGCCGCGGTCTGGGTGCCGCCTCGGCCGCGGCCGCTCTCCCGCGCTCCTTTGCCA

-445 GAAGGTAATCTCCGTGAACAGGGGAGGGAGGCGAGCAGGGAGGAAGGAGGGGTGGCCAGGAGGAAGGGGGG

-374 CGTGGGGAGGCGGCTTTTCTCTCTCCCTCTCTCCCTTTCCAAATGATTCAGAAGTCGATAAGACCAGGAGA

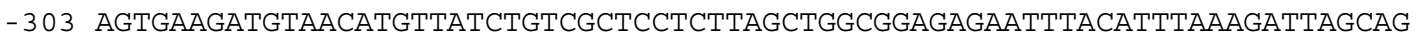

-232 AGTGAGAAAGAGAAATCTGCCTTTTGTTGTGTGGGGTGAGGAGGAGGCATCTACCCCTGGCCTTGACGCTA

- 161 TCTCCCATCACCTCTGCTATCCAGACAGGACTCACCGAGGTGAGAATACCGGAGGGCCTTATCTTTAATTG

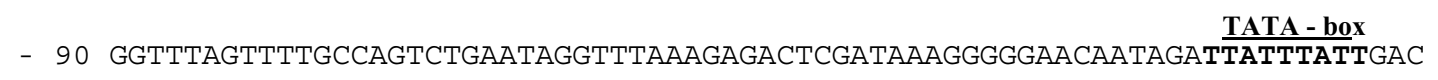 \\ - 19 TGgACGCTGAAGCCTTTAgAtgAagA \\ $+1$
}

Figure 1 The sequence of the 5 'flanking region of ETO. (A) Alignment with genomic DNA of ETO cDNA from HEL cells amplified by RLM-RACE. Nucleotide +1 indicates the translational start site (ATG). The transcription start site is shown to be at $-318 \mathrm{bp}$. (B) The sequence of the 5 'flanking region of the ETO promoter region was amplified by PCR with genomic DNA as template. Putative consensus binding sites for transcription factors identified with MatInspector http://www.genomatix.de/matinspector.html are marked. Alignment of the human sequence with mouse and rat genomic sequences showed a region at -659 to $-432 \mathrm{bp}$ (underlined) to be highly conserved suggesting the presence of a proximal promoter. 


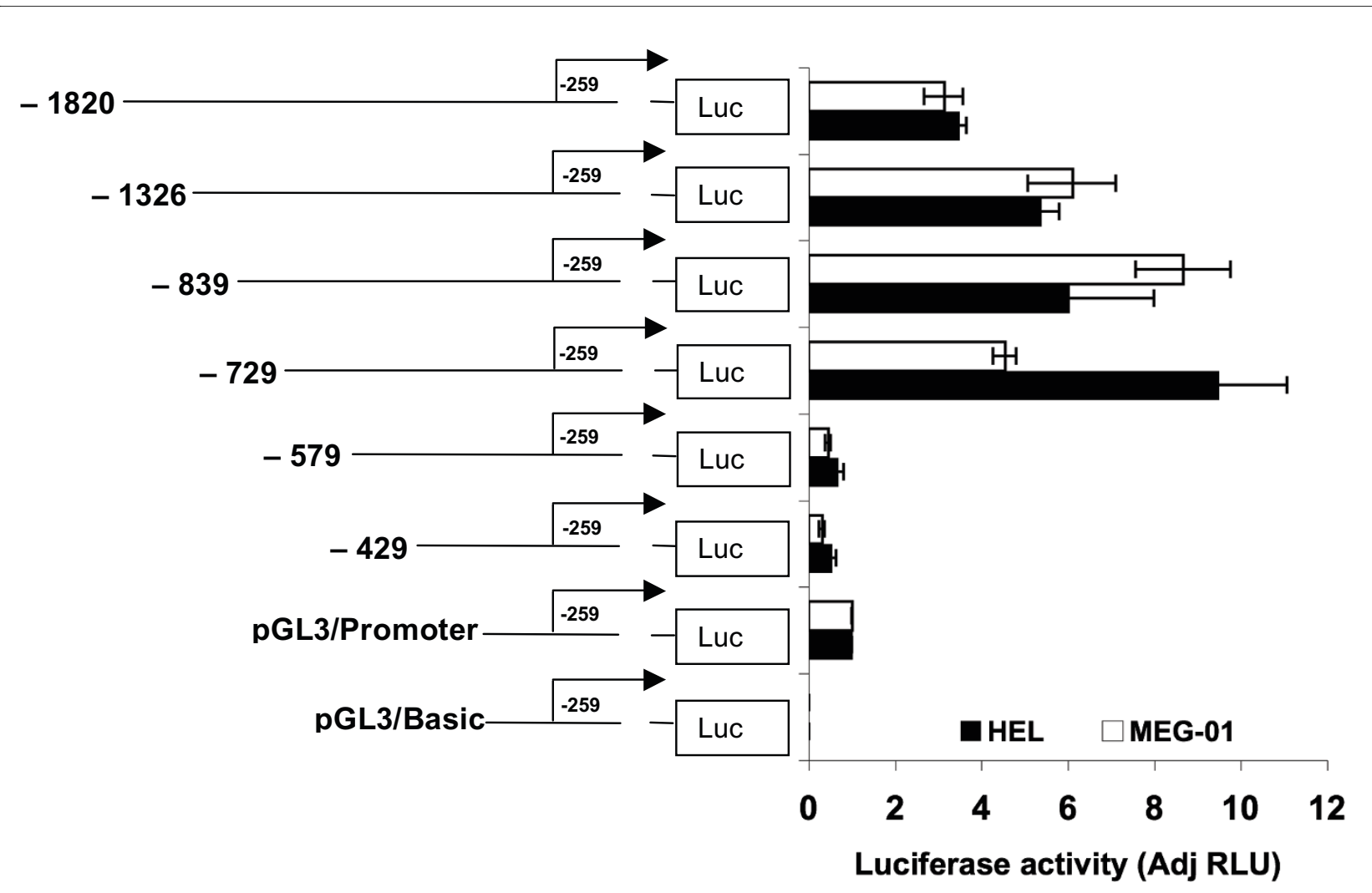

Figure 2 Effects of 5'deletions on ETO promoter activity in erythroleukemia HEL and megakaryocytic MEG-01 cells. The following reporter constructs were examined: pGL3 -1820-259 (-1820), pGL3 -1326-259 (-1326), pGL3 -839-259 (-839), pGL3 -729-259 (-729), pGL3 -579-259 (-579), and pGL3 -429-259 (-429) after transfection into erythroid (HEL) and megakaryocytic (MEG-01) cell lines. Nucleotide +1 indicates the translational start site (ATG) and nucleotides 5'and 3' thereof have a "-" and "+" designation. The promoterless pGL3/basic and the pGL3/SV40-promoter are used as negative and positive control, respectively. Firefly and Renilla luciferase (internal standard) activities were assayed $24 \mathrm{~h}$ post-transfection. The luciferase activity is normalized against pGL3/SV40-promoter activity. The transcriptional activity of the full-length promoter was retained by the -729 to $-259 \mathrm{bp} \mathrm{region}$, which is therefore likely to contain the proximal ETO promoter. The -579 to $-259 /-429$ to -171 bp regions showed lack of transcriptional activity. Firefly was normalized to Renilla luciferase as internal control for transfection efficiency and the results are given as adjusted Relative Luciferase Units (AdjRLU). Bars represent the mean of results from 3 to 5 separate transfections and the error bars show SEM.

The -579 to -259 and the -429 to -259 bp regions obtained by further deletions showed no transcriptional activity and may not play a significant role in ETO gene expression in HEL or in MEG-01 cells. Hence, the -729 to -259 bp region represents the smallest fragment generated herein that retained full transcriptional activity. Thus, the results from both phylogenetic footprinting and deletional analyses reveal a region, which is likely to contain the proximal ETO promoter.

The likely proximal ETO promoter region (-729 to -259 bp) was investigated for cell specificity. The pGL3 -729259 reporter plasmid gave a strong signal in erythroid (HEL) and megakaryocytic (MEG-01) cell lines and a low signal in promyelocytic HL-60, myelomonocytic U-937 and monkey kidney COS-7 cells (Fig. 3). As shown by results from real-time PCR, ETO transcripts were detected in the erythroid/megakaryocytic cell lines but not in the myeloid cell lines U-937 and HL-60 or in COS7 cells (Fig. 3). Thus, even if a limited number of cell lines were investigated a robust correlation is suggested between transfected promoter activity and endogeneous ETO gene activity. This result suggests cell-type-specific activation of the ETO promoter. However, transfected promoter activity did not correlate directly with the ETO mRNA levels detected with real-time PCR. For example, MEG-01 cells showed higher luciferase activities than HEL cells but lower transcript levels (Fig. 3). The relationship between promoter activity and mRNA activity is affected by the endogeneous environment. Gene regulation involves more than promoter activity. Differences in repressor elements could explain the lack of correlation between promoter activity and mRNA. 
Ajore et al. BMC Molecular Biology 2010, 11:38

Page 5 of 15

http://www.biomedcentral.com/1471-2199/11/38

$\begin{array}{lllll}\text { HEL } & \text { MEG-01 } & \text { U-937 } & \text { HL-60 } & \text { COS -7 }\end{array}$
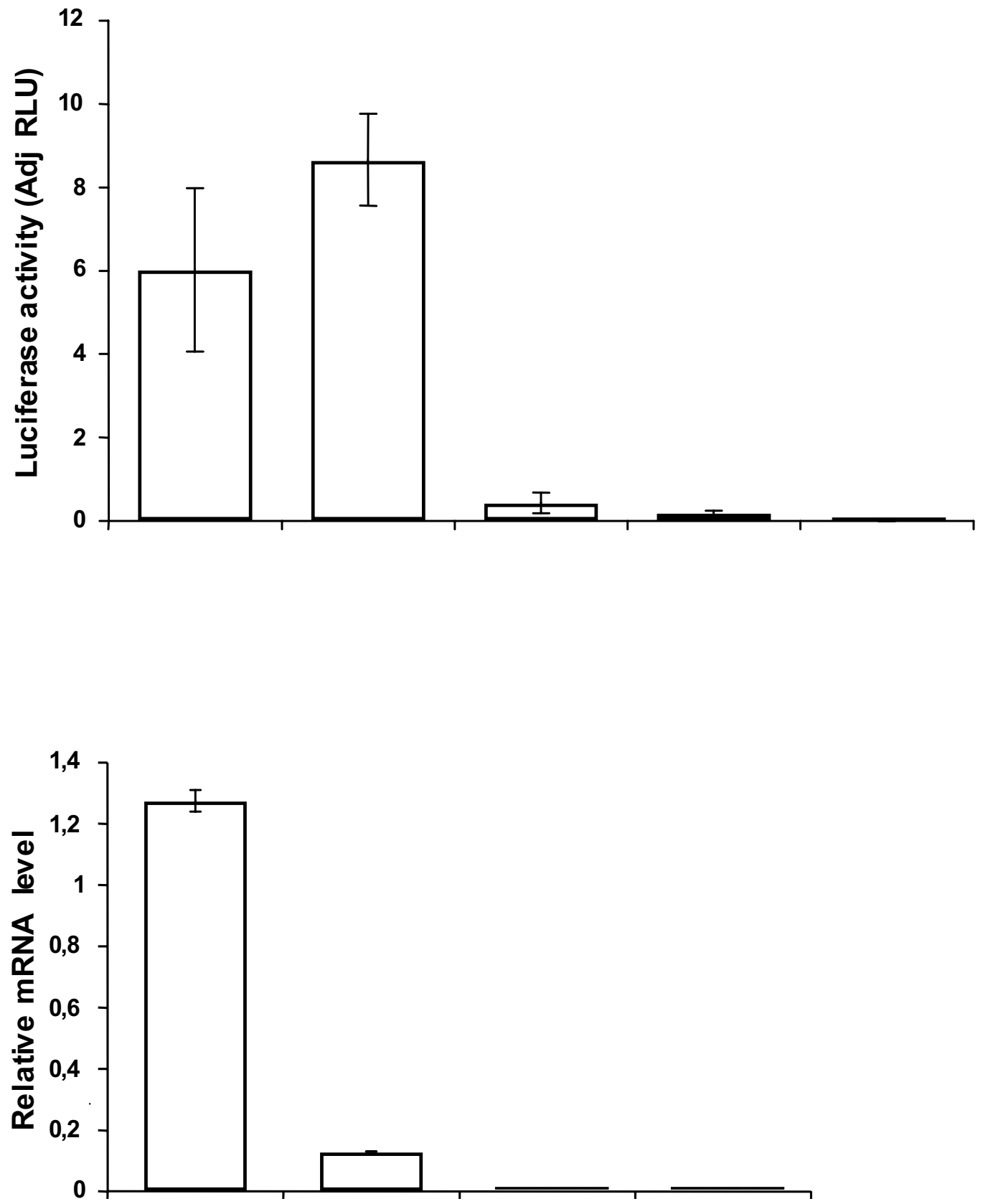

Figure 3 Relative activity of the proximal ETO promoter (-729 to $-\mathbf{2 5 9} \mathbf{b p}$ ) reporter expressed in various cell lines. The pGL3/basic and pGL3/ SV40-promoter are used as negative and positive control, respectively. The luciferase activity is normalized against pGL3/promoter activity. The ETO729 to -259 bp region reporter shows strong activity in the erythroleukemia HEL and in the megakaryocytic MEG -01 cell lines. The myelomonocytic U-937, the promyelocytic HL-60 and the monkey kidney COS-7 cell lines show only low luciferase activity upon expression of ETO -729 to -259 bp regin. ETO transcripts detected by real-time PCR were found in cell lines showing increased luciferase expression upon transfection of the ETO -729 to -259 bp region. Lack of ETO expression in COS-7 cells was shown before [54]. Firefly was normalized to Renilla luciferase as internal control for transfiction efficiency and the results are given as adjusted Relative Luciferase Units (AdjRLU). Luciferase results are shown for 3 to 5 separate transfections; bars represent the mean and the error bars show SEM. Real-time PCR results are from two experiments in triplicate samples. Relative luciferase unit (RLU) represents experimental value for luciferase activity. 
Mutagenesis of the GATA - 636 consensus binding site represses and mutagenesis of the ETS1 -705 binding site increases transactivation of the ETO promoter in HEL/MEG01 cells

As mentioned above several potential transcription factor binding sites were detected on the conserved region of the promoter (Fig 1). ETS and GATA factors play a role in erythroid differentiation and CAAT-binding sites are often involved in promoter regulation. Therefore, we choose to determine whether the identified potential transcription factor binding sites of the conserved region contribute to transactivation of the promoter each element was disrupted by site-directed mutagenesis (Fig. 4). Disruption of the GATA -636 binding site led to a 4-fold reduction in reporter gene activity in HEL/MEG-01 cells relative to intact ETO promoter. Conversely, mutation of the ETS1 -705 binding site (outside the evolutionary conserved region) increased the luciferase signal twice. Disruption of the GATA -651, GATA -619, ETS1 -661, and the CAAT -633 binding sites did not significantly affect the reporter signal. CAAT -633 was mutated because CAAT plays a role in promoter regulation. The results suggest that the GATA -636 site may have a role in activation and that the ETS1 -705 sites may have a role in repression of the ETO gene activity in cells with erythroid/megakaryocytic potential.

\section{GATA-1 binds to consensus sites in the ETO promoter in vitro and in vivo of HEL/MEG-01 cells but not in in vitro in G1E cells}

Electrophoretic Mobility Shift Assays (EMSA) and antibody supershift assays were used to examine interactions of the putative GATA binding sequences (GATA -651, GATA -636 and GATA -619) using nuclear extracts from HEL or MEG-01 cells. Biotin-labeled probes, which include the various GATA binding sequences were used for EMSA. Binding of proteins from nuclear extracts to biotinylated probe that includes the GATA -636 sequence was indicated by gel shift (Fig. 5). Specificity of the shift was shown by lack of binding to a probe with mutations within the core consensus sequence and by inhibition of binding of biotinylated probe by excess unlabeled probe. Proteins bound to the GATA -636 probe were "supershifted" by antibody to GATA-1 but not by antibody to GATA-2 indicating binding of GATA-1 to the consensus site (Fig. 5). GATA -619 and -651 probes also showed a shift that was competed for by excess unlabeled probe indicating specific binding of nuclear extract protein (Fig.

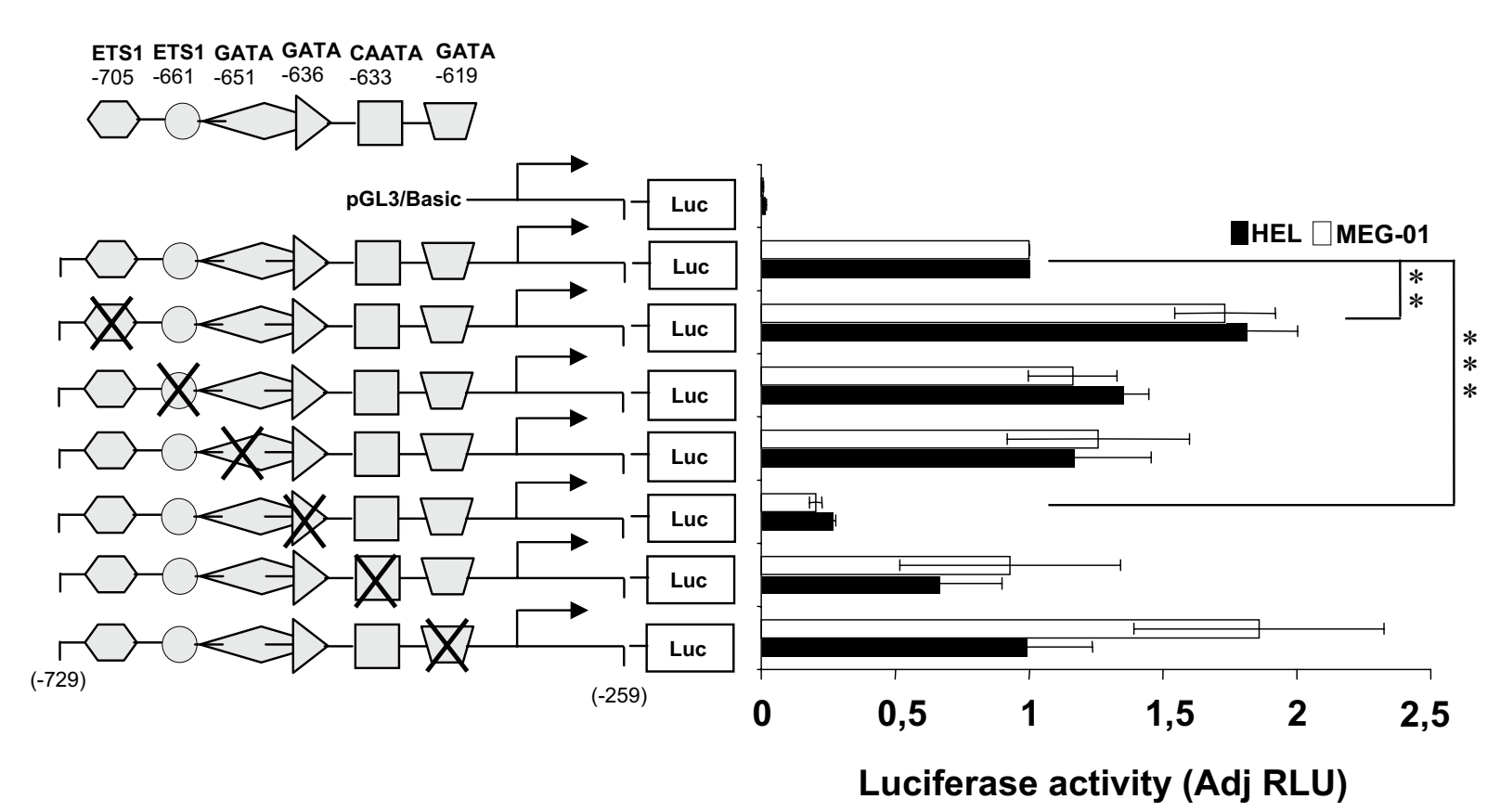

Figure 4 Mutagenesis of consensus transcription factor binding sequences in the 5'ETO. Erythroid HEL and megakaryocytic MEG-01 cells were transfected with the -729 to -259 bp ETO promoter reporter construct with or without mutations of transcription factor binding sites as indicated (X). The mutations are described in "Materials and Methods". The pGL3/basic and pGL3/SV40-promoter are used as negative and positive control, respectively. Firefly and Renilla luciferase (internal standard) activities were assayed $24 \mathrm{~h}$ post-transfection. The luciferase activity of mutated ETO promoter is normalized against luciferase activity of wildtype ETO promoter. Mutation of the GATA -636 site strongly represses transactivation of the ETO promoter reporter. Mutation of the ETS1 -705 binding site increased the luciferase signal twice. Firefly was normalized to Renilla luciferase as internal control for transfection efficiency and the results are given as adjusted Relative Luciferase Units (AdjRLU). Bars represent the mean of results from 3 to 5 separate transfections and the error bars show SEM. **, $p<0.01 ; * *, p<0.0001$ 

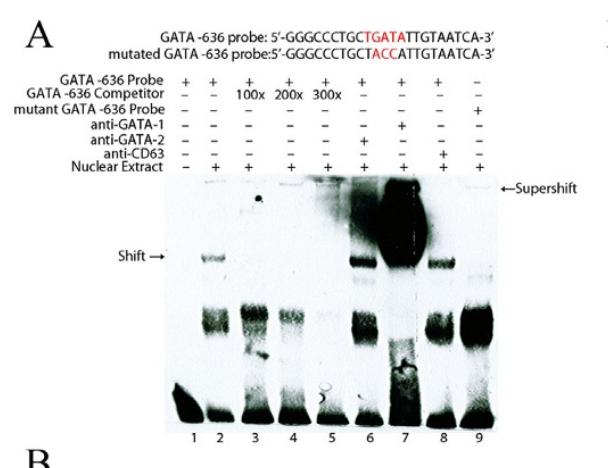

B

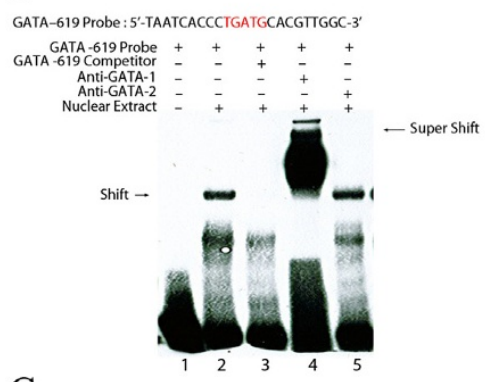

C

GATA -651 Probe: 5'- TTCCTGCCTCCATCTGGGCCCTG-3'

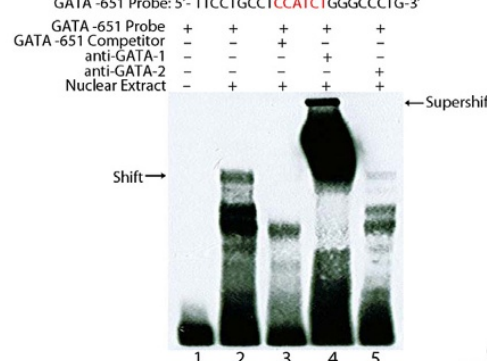

GATA -636 Probe
D

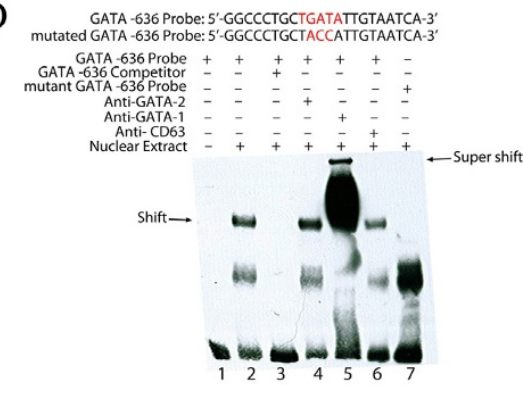

E

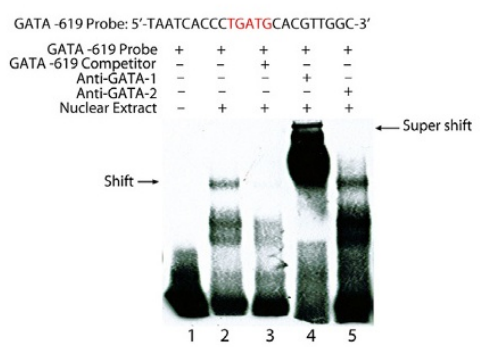

F

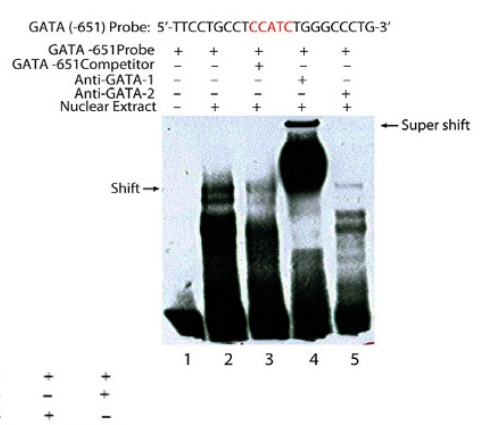

G G1E-2 Nuclear Exract

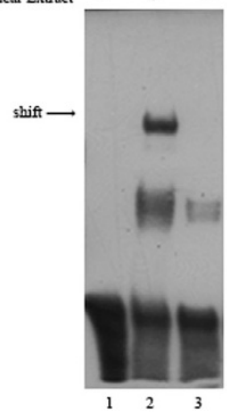

Figure 5 Detection of DNA-protein interactions using electrophoretic mobility shift/supershift assays in vitro of consensus GATA binding sequences in the 5'promoter of ETO and nuclear extracts from HEL/MEG-01/G1E cells. Sequences for oligonucleotide probes of core consensus and mutated GATA -651, GATA -636 and GATA-619 sites are shown. Arrows marked shift demonstrate primary DNA-nuclear protein interactions; arrows marked supershift demonstrate DNA-nuclear protein-antibody interactions. Results for HEL cells (A-C) are to the left, results for MEG-01 cells (D-F) to the right and results for the G1E (G) is at bottom. For the GATA -636 probe a shift is shown in HEL cells (A2) that is competed for by excess unlabelled probe (competitor) (A3-A5) indicating binding of nuclear extract protein to the biotinylated probe that contains the GATA -636 sequence. In support of this no binding was observed to a probe that contains a mutated GATA -636 sequence (A9). Proteins bound to the GATA -636 probe were "supershifted" by antibody to GATA-1 (A7) but not with antibody to GATA-2 (A6) indicating specificity of the DNA-protein interaction. Similar results are shown for the GATA -636 probe in MEG-01 cells (D). For GATA -619 and -651 probes a shift is shown (B, C, E, F; lane 2) that is competed for by excess unlabeled probe (competitor) indicating binding of nuclear extract protein to the biotinylated probe that contains GATA -619 or - 651 sequences. A supershift is shown with antibody to GATA-1 ( $B, C, E$, F; lane 4) but not with anti-GATA-2 (B, C, E, F; lane 5). To try to distinguish between the binding of GATA-1 and GATA-2 to the ETO promoter EMSA was performed with nuclear extract of G1E cells, which lack GATA-1. MEG-01 nuclear extract was used as positive control (G2). No binding of GATA-2 protein to the consensus ETO promoter was observed (G3) suggesting lack of GATA-2 interaction. These experiments were repeated twice. 
5). Furthermore, proteins bound to the GATA -651 and GATA -619 elements were "super-shifted" by antibody to GATA-1 but not by antibody to GATA-2 (Fig. 5). This indicates that GATA-1 can bind to all three GATA consensus sites within the conserved region of the ETO promoter.

The lack of GATA-2 binding to the -636 probe could result from competition from GATA-1. To determine whether GATA-2 binding can be competitively inhibited by GATA-1, EMSA was performed with nuclear extract of the G1E cell line, which is GATA-1- (null) but GATA-2+ [27]. No primary interaction of GATA-2 with probe that included the GATA - 636 sequence was seen (Fig 5G, Lane $3)$. In lack of primary GATA-2 protein-DNA interaction no supershift was observed with anti-GATA-2 (not shown). The results argue against strong binding of GATA-2 to the -636 site, and therefore do not support competition from GATA-1 for GATA-2 binding, although this can not be entirely ruled out for the experiments with MEG-01/HEL cells.

An additional band besides the one labeled "shift" is present in the EMSA experiments of Fig. 5. It is still present with the GATA mutant oligo (Fig 5A, lane 9) indicating that it is non-specific, but the band is lost by supershift (Fig 5A, lane 7), suggesting that it contains GATA-1 protein. It may represent a non-specific GATA-1 interaction.

Chromatin immunoprecipitation (ChIP) assays were used to examine in vivo binding of GATA-1 and GATA-2 to the putative ETO gene promoter. ChIP assays were performed using chromatin isolated from HEL or MEG01 cells and antibodies towards GATA-1 or GATA-2. The presence of GATA-1 and GATA-2 in HEL and MEG-01 cells was confirmed by Western blotting (Fig. 6). The precipitated DNA was examined by PCR amplification of the ETO promoter fragment using gene specific oligonucleotides. By using primers specific for the evolutionary conserved region of the ETO promoter, PCR products of 90 bp were generated from the anti-GATA-2- and antiGATA-1-immunoprecipitated chromatin of MEG-01 cells (Fig 6, top, lanes 6 \& 11). ETO promoter amplification with anti-GATA-2 was also obtained in HEL cells (Fig 6, bottom, lane 6). ChIP with anti-GATA-2 and amplification of control region with primers $B$ was negative in both MEG-01 (top, lane 7) and HEL cells (bottom, lane 7) and amplification with control primers B was also negative with anti-GATA-1 in both HEL and MEG-01 cells (not shown). ChIP controls with anti-actin or without antibody were negative (both panels, lane 2-5), while positive PCR controls with genomic DNA were positive (both panels, lanes 8-9. Thus, specific amplification was achieved by precipitation with anti-GATA-1 or antiGATA-2 only. No amplification was seen in the absence of antibody or in the presence of anti-actin antibody.
In conclusion, results from EMSA/supershift assays demonstrate GATA-1 binding in vitro to the GATA -636 binding site supported by ChIP assays demonstrating binding in vivo of GATA-1 to the putative ETO promoter. These results are consistent with a function of GATA-1 in activation of the ETO promoter suggested by the results of the mutagenesis studies depicted in Fig. 4.

\section{Overexpression of GATA-1 stimulates the ETO promoter}

GATA-1 was transiently overexpressed in HEL/MEG-01 cells to determine the effect on co-transfected ETO -729 to $-259 \mathrm{bp}$ promoter. The ETO promoter was stimulated in a dose-dependent manner by GATA-1 (Fig. 7A). This result is consistent with a role of GATA-1 in transactivation of the promoter.

\section{Expression of AML1-ETO represses the ETO gene reporter in HEL/MEG-01 cells}

AML1-ETO was transiently expressed in HEL/MEG-01 cells to determine the effect on the co-transfected ETO 729 to -259 bp proximal promoter reporter. The ETO promoter reporter was strongly repressed in a dosedependent manner by expression of AML-ETO (Fig. 7B)

\section{Discussion}

The goal of this work was to feature the promoter of the ETO co-repressor gene. To this end, we identified essential cis-acting elements and trans-acting factors that govern ETO expression within hematopoietic cells. We identified the likely proximal promoter of the ETO gene whose expression within hematopoiesis seemed to be restricted to erythroid/megakaryocytic cells. Examination for regulatory cis-elements of a $1.5-\mathrm{kb}$ region upstream of the transcription start site of the 5 ' flanking region of the ETO gene revealed an initial $400 \mathrm{bp}$ stretch to be required for maximal ETO promoter reporter signal when examined in erythroid/megakaryocytic cell lines, which have endogeneous ETO expression. Conversely, the ETO promoter gave no reporter signal when examined in hematopoietic cell lines with lack of endogeneous ETO. Phylogenetic footprinting revealed a $196 \mathrm{bp}$ region at -659 to $-462 \mathrm{bp}(+1$ indicates translational start codon) containing cis-acting elements with GATA binding sites required for regulation of ETO transcription.

Disruption of the GATA - 636 site within the conserved region repressed transactivation whereas disruption of the ETS1 -705 binding site activated the ETO promoter. Examination in vitro with EMSA revealed binding of GATA-1 but not of GATA-2 to a probe that included GATA -636 site sequences, the disruption of which abolished transactivation of the ETO gene. Our demonstration that EMSA from the G1E cell line, which expresses GATA-2 but lacks GATA-1, also did not show GATA-2 binding to the -636 site, suggests that GATA-2 does not 


\author{
GATA-1 $\rightarrow-$ \\ GATA-2 $\rightarrow=$
}

Lamin B1
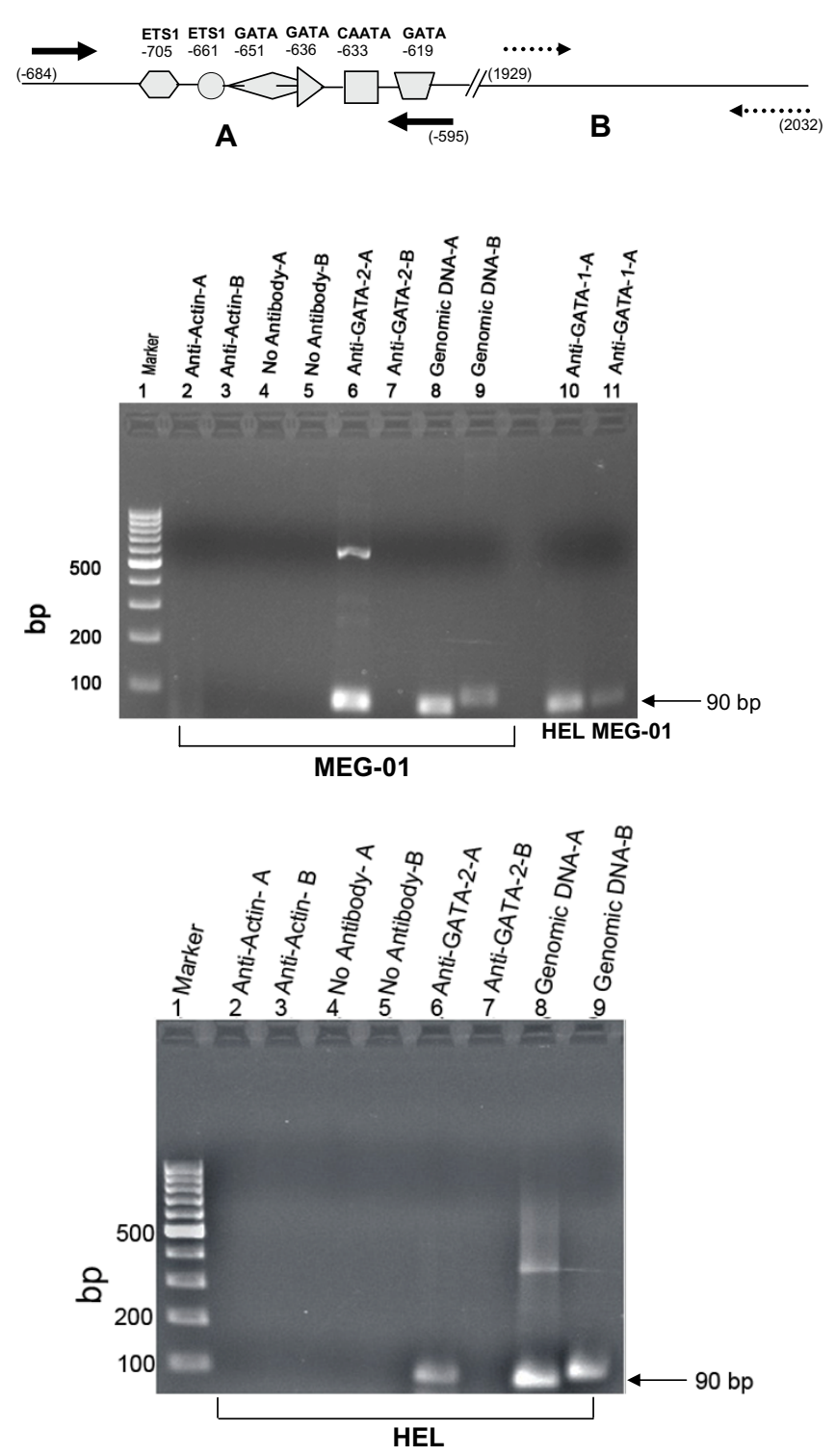

Figure 6 Chromatin immunoprecipitation (ChIP) assay for examining interactions in vivo of consensus GATA binding sequences in the 5'promoter of ETO. The forward and reverse primers used to amplify the proximal promoter region from -684 to -595 (primers A, solid arrows) and forward and reverse primers for a downstream region from 1929 to 2032 as control (primers B, dashed arrows) are shown. ChIP assays were carried out as described in Methods using chromatin isolated from HEL and MEG-01 cells. PCR products were separated on a 2\% gel and representative results are shown. Top and bottom gel figures represent MEG-01 and HEL cells, respectively, except that lane 10 in top gel represents HEL cells. Lane 1, 100bp ladder; lane 2, actin antibody and primers $\mathbf{A}$; lane 3, actin antibody and primers $\mathbf{B}$; lane 4, no antibody and primers $\mathbf{A}$; lane 5, no antibody and primers B; lane 6, GATA-2 antibody and primers A; lane 7, GATA-2 antibody and primers B; lane 8, genomic DNA and primers $\mathbf{A}$; lane 9, genomic DNA and primers B. GATA-1 precipitated chromatin amplified with primers $\mathbf{A}$ in HEL and MEG-01 cells is shown in lane 10 and lane 11, respectively (top gel). By using primers specific for the evolutionary conserved region of the ETO promoter, a PCR product is generated both from the anti-GATA-1 and the antiGATA-2 immunoprecipitated chromatin. No amplification is seen in the absence of antibody or in the presence of anti-actin. The experiment was repeated twice. 
A

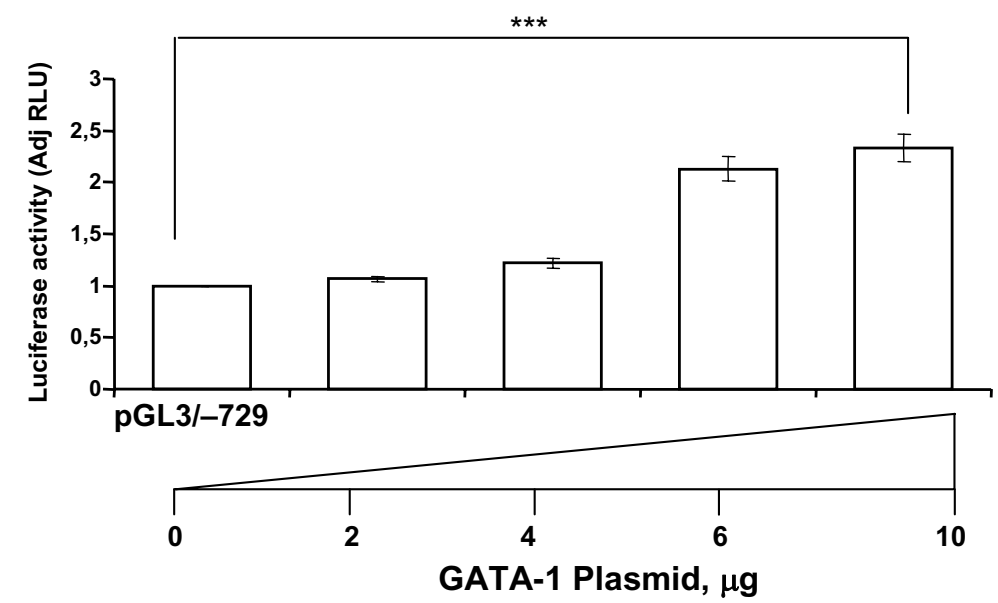

B
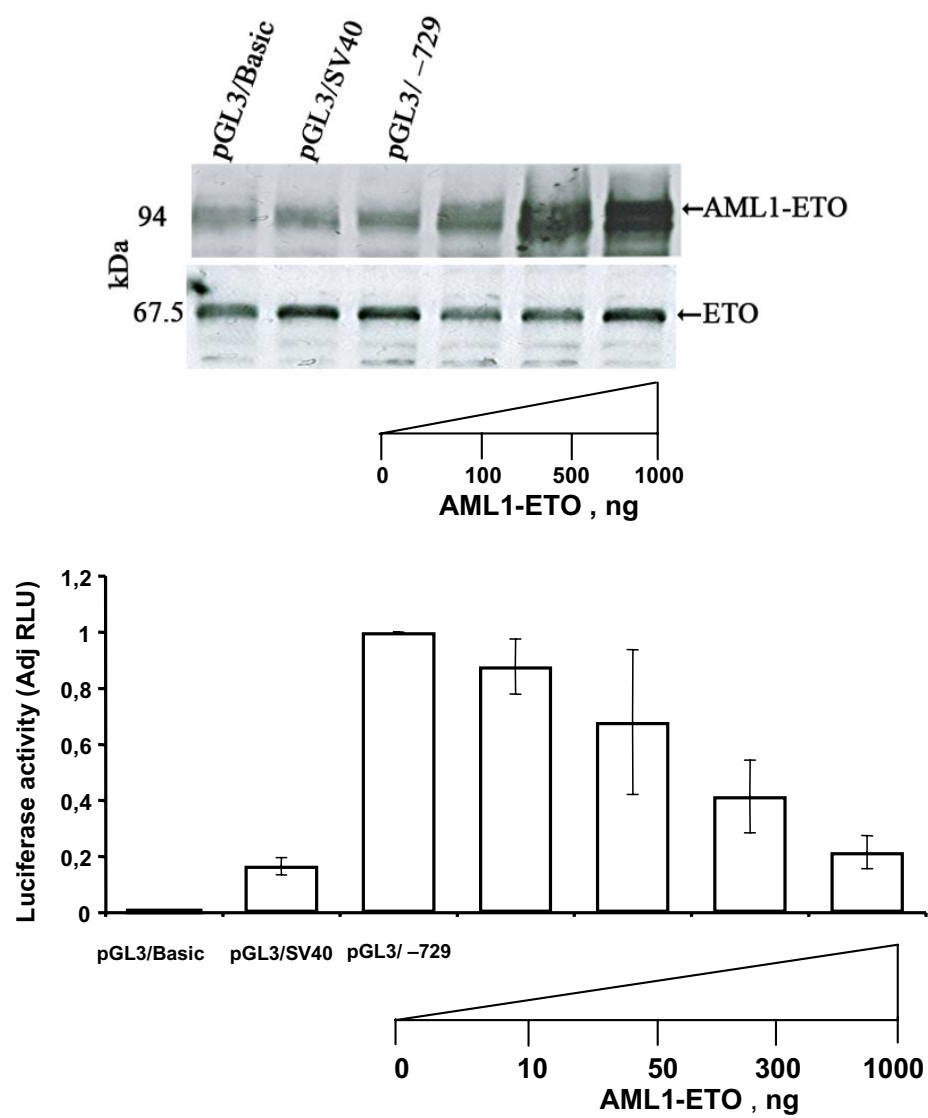

Figure 7 Effects of overexpression of GATA-1/GATA-2 or AML1-ETO on the ETO promoter reporter. (A) HEL/MEG-01 cells were co-transfected with $15 \mu \mathrm{g}$ ETO -729 to -259 bp promoter plasmid and 0 to $10 \mu \mathrm{g}$ of GATA-1 plasmid. The luciferase activity is normalized against the ETO -729 to -259 promoter. The ETO promoter is activated by overexpression of GATA-1 in a dose-dependent manner. Similar results were obtained by co-transfection of HEL cells (data not shown). (B) MEG-01 cells were co-transfected with $15 \mu \mathrm{g}$ ETO-729 to -259 bp promoter plasmid and 0 to $1 \mu \mathrm{g}$ of AML1-ETO plasmid. The $\mathrm{pGL} 3 /$ basic and $\mathrm{pGL3/SV40-promoter} \mathrm{are} \mathrm{used} \mathrm{as} \mathrm{negative} \mathrm{and} \mathrm{positive} \mathrm{control,} \mathrm{respectively.} \mathrm{The} \mathrm{luciferase} \mathrm{activity} \mathrm{is} \mathrm{normalized} \mathrm{against}$ ETO -729 to -259 bp promoter. The ETO promoter is strongly repressed in a dose-dependent manner by AML-ETO. Western blotting shows exogeneous AML1-ETO (detected with anti-MTG) and endogeneous ETO expression (detected with anti-ETO). These experiments were repeated three times with similar results. Firefly was normalized to Renilla luciferase as internal control for transfection efficiency and the results are given as adjusted Relative Luciferase Units (AdjRLU). ${ }^{* *}, p<0.0001$ 
bind to this site. However, the lack of binding of GATA-2 to this probe is of uncertain significance and it is not possible to definitely distinguish between GATA-1 and GATA-2 interactions at the ETO promoter. Examination with ChIP assay revealed binding in vivo of GATA-1 to elements within the conserved region of the ETO promoter. Furthermore, the promoter was stimulated by overexpression of GATA-1. Collectively, our results demonstrate that the GATA-1 transcription factor binds to the ETO proximal promoter and is involved in ETO gene expression. The GATA-1 transcription factor is a master regulator in erythroid/megakaryocytic development $[28,29]$.

\section{Role of GATA-1/ETO in hematopoiesis}

GATA-1 belongs to a family of GATA transcription factors, which bind to DNA sequences within the internal GATA-motif A/T(GATA)A/G [30]. GATA-1/GATA-2 recognize similar DNA-binding motifs; their expression profiles overlap for example in the erythroid lineage. GATA-1-mediated ETO activation is in agreement with GATA-1 being a critical direct repressor of several target genes including GATA-2 [31,32], the repression of which facilitates erythroid differentiation [31,33]. In addition to the cis-acting GATA elements, a putative ETS1 binding element was also identified within the conserved region of the ETO proximal promoter and shown to mediate suppressor activity. Many members of the ETS family for example PU.1, Fli1 and ETS1 are known to play an important role in megakaryocytic and erythroid differentiation [34].

The GATA family of transcription factors contains important regulators of gene expression in hematopoietic cells $[35,36]$. GATA-1 is essential for the development of early and definitive erythropoiesis/thrombopoiesis $[28,29]$. GATA-1 deletion results in blocked terminal erythroid and megakaryocytic maturation [37-39]. GATA-1 and GATA-2 are expressed reciprocally during erythropoiesis, GATA-1 levels rise when GATA-2 levels decrease [40]. What role does ETO have in erythroid/ megakaryocytic development and differentiation? The regulation of the ETO promoter by GATA-1 suggests a role of ETO-mediated gene suppression at a phase of erythropoiesis/thrombopoiesis when GATA-1 is up $[41,42]$. GATA-1 is expressed at high levels during terminal maturation of erythroid/megakaryocytic cells [43]. Thus, ETO-mediated gene suppressor action may have a role during terminal erythroid/megakaryocytic maturation as a result of GATA-1-mediated ETO transactivation.

GATA-1 has a role in erythroid/megakaryocytic cell proliferation and differentiation [44] by activating erythroid-specific genes [33] or megakaryocyte-specific genes $[38,39,45]$ and repressing genes associated with prolifera- tion $[31,33,46,47]$. The expression level of one member of the ETO homologues, murine MTG16 (ETO2) has already been shown to regulate expansion of erythroid progenitors [3]. Likewise, ETO2 expression in megakaryocytic cells is restricted to immature megakaryocytes and restrains their differentiation [48]. Therefore, ETO2 is suggested to repress inappropriate early expression of terminal megakaryocyte genes by binding to GATA-1 [48]. We have observed that MTG16 decreases during early in vitro-induced human erythropoiesis whereas ETO is increased transiently during the peak of erythropoiesis [19]. Therefore, it is possible that ETO, in contrast to MTG16 (ETO2), has a role in repressing genes associated with self renewal and proliferation and that GATA1-activation of the ETO gene might be viewed in this context.

\section{ETO homologue functions}

The ETO homologues are expressed in hematopoietic cells in a more or less cell-type-specific manner [19]. This is supported by the present work, which indicates differences in promoter regulation among the ETO homologues as a possible explanation for lineage-specific expression. We find that the ETO promoter is regulated by cis-acting elements contained within an evolutionary conserved region, which is lacking in the $5^{\prime}$ flanking region of both MTG16 and MTGR1 (in silico, data not shown). The 5' flanking region of MTGR1 contains an evolutionary conserved region lacking in the two other ETO homologues (in silico, data nor shown). The celltype-specific hematopoietic expression of ETO is much tighter than that of MTG16 and MTGR1 suggesting specific ETO functions. However, even though their genes are differently regulated, the ETO homologues could have redundant functions if they are expressed in the same cell-type-specific context.

\section{Suppression of ETO promoter by AML1-ETO}

The AML1-ETO fusion protein, which is a gene product of the $(8 ; 21)$ chromosomal translocation of acute leukemia $[14,15]$, binds the promoter region of many genes mostly causing transcriptional suppression [49]. However, some genes regulated by AML1-ETO do not show binding of the fusion protein to the promoter, the transactivation of which is instead affected indirectly $[49,50]$. The AML1-ETO-mediated suppression of the ETO promoter observed is unlikely to be due to a direct competition for AML1-binding sites, which are not detectable on the promoter. Nevertheless, our observation may be relevant to the reported AML-ETO-induced block of erythroid development $[23,24]$. If ETO is normally involved in repressing genes associated with self renewal and proliferation, suppression of the ETO gene by AML1-ETO 
could facilitate the AML1-ETO-induced block of erythroid lineage commitment.

\section{Conclusion}

In conclusion, we report that the GATA-1 transcription factor binds to the ETO proximal promoter and transactivates the gene in cells of erythroid/megakaryocytic potential in a cell-type-specific manner. The same transacting factors that are essential in ETO expression are essential in erythroid/megakaryocytic differentiation.

\section{Methods}

\section{Cell culture}

The human myelomonocytic U-937, human erythrolukemic HEL, megakaryocytic MEG-01, and promyelocytic HL-60 cell lines were maintained in RPMI-1640 medium supplemented with 10\% Fetal Bovine Serum (FBS) (Gibco BRL, Life Technologies, Rockville, MD, USA). Monkey kidney COS-7 cells were maintained in DMEM medium with $10 \%$ FBS supplemented with high glucose $(4.5 \mathrm{~g} / \mathrm{l})$ and L-glutamine. The G1E erythroid cell line, derived from murine embryonic stem cells [27], was maintained in IMDM, Pen/Step $20 \mathrm{ml} / \mathrm{L}$, monothioglycerol $12 \mu \mathrm{l} / \mathrm{L}$, FCS 20\%, human erythropoietin $2 \mathrm{U} / \mathrm{ml}$, Stem Cell Factor $50 \mathrm{ng} / \mathrm{ml}$. GIE cells express GATA-2 mRNA at a high level relative to the expression in wild-type proerythroblasts or erythroleukemia cells but are GATA-1-(null) [27].

\section{5'-rapid amplification of CDNA-ends (RACE)}

The mRNA was prepared from HEL cells using Oligotex Direct mRNA mini Kit (Qiagen, Hilden, Germany). The 5 '-end (transcription start) of the mRNA was identified by the first choice RNA Ligase mediated (RLM)-RACE kit (Ambion Inc., TX, USA). Nested PCR of the RACE reaction was performed with adapter primer 5'-CGCGGATCCGAACACTGCGTTTGCTGGCTTTGATG-3' and nested gene specific primer 5'-ACTGGTTCTTGGAGCTCCTTGAGTAGT-3'. RACE- products were cloned into pGEM-T Easy Vector system (Promega Corporation, WI, USA) and sequenced.

\section{Amplification of ETO promoter region}

A 5 ' flanking region of $2049 \mathrm{bp}$ from -2307 to $-259 \mathrm{bp}$ was amplified from human genomic DNA by PCR. The forward and reverse primers used were 5'-TGGAGAAATATTACCTTGTTCTCGTCTCAG-3' and 5'-ACACAA CAAAAGGCAGATTTCTCTTTCTCAC-3', respectively. Regions corresponding to -1820 to $-259,-1326$ to 259 and -839 to -259 bp were amplified from the $2049 \mathrm{bp}$ fragment by nested PCR with forward primers 5'TAGCTCGGTACCACTTTCTGGCCCCATCC-3' (KpnI restriction site highlighted in bold),5'- AGCAGGTACCTAAGAGTCACCTGTGGCTAC-3' (KpnI restriction site highlighted in bold), 5'-ACAGCAGCTAGCGCTACAGTGCACACCATG-3' (Nhe I restriction site highlighted in bold) and a common reverse primer $5^{\prime}$-TC CGCCAGATCTGAGGAGCGACAGATAAC-3' (BglII restriction site highlighted in bold). Sequential 5 ' deletions of the -1820 to -259 bp promoter region were generated by PCR from the cloned genomic DNA as template to generate -729 to -259 , the -579 to -259 and the -429 to -259 bp regions. Forward primers were 5'-AACAGGTACCGGAGGCGAGCAGGGAGG-3', 5'-ACACGGTA CCACACACAATGTGCCATCCTG-3' and 5'-TGT CTG GTACCTCTCTCTCССАСТTTTCC-3' with KpnI restricton sites highlighted in bold. The common reverse primer is the same as used for amplification of the -2049 to $-259 \mathrm{bp}$ region. All sequences were verified.

\section{Site-directed mutagenesis of transcription factor-binding sites}

Oligonucleotide primers including desired mutations were synthesized and used in two-step spliced overhang extension PCR. The following potential transcription factor binding sites were mutated: ETS1 sites at positions 705 and $-661,5$ '- TCC-3' changed to 5'-GAA-3'; GATA site at position -651, 5'-ATC-3'changed to 5'-GCT-3'; GATA site at position -636, 5'-GAT-3' changed to 5'ACC-3'; CAAT site at position -633, 5'-ATT-3' changed to 5'-GCC-3'; GATA site at position -619, 5'-GAT-3' changed to 5 -AGC-3'. After subcloning into promoterless pGL3/Basic reporter plasmid, the mutations were verified by sequencing.

\section{Luciferase reporter assays}

PCR products were cloned into promoterless pGL3/Basic reporter plasmid employing firefly luciferase as specific reporter to generate pGL3 -1820-259, pGL3 -1326-259, pGL3 -839-259, pGL3 -729-259, pGL3 -579-259, and pGL3 -429-259 reporter constructs. The mutants were cloned into the same reporter plasmid. Transient transfections of hematopoietic cell lines were performed by electroporation as previously described by Lennartsson et al [51]. The pGL3/SV40-promoter vector served as positive control and promoterless pGL3/Basic vector as negative control. Renilla luciferase was used as internal control for transfection efficiency. Thirtyfive $\mu \mathrm{g}$ pGL3 DNA were used for HL-60 target cells and $15 \mu \mathrm{g}$ for U937, HEL or MEG-01 target cells. At $24 \mathrm{~h}$ after transfection cells were disintegrated in $200 \mu \mathrm{l}$ lysis buffer. Twenty $\mu \mathrm{l}$ triplicate lysate samples were used for luciferase assays with the Dual luciferase reporter assay kit (Promega Corporation, WI, USA). Onehundred $\mu \mathrm{l}$ each of firefly and Renilla substrates were added. Light emission was quantified using standard procedures (Run Promega Protocol, DLR-0-INJ) on the GLOMAX 20/20 Luminometer. Firefly was normalized to Renilla luciferase as internal con- 
trol for transfection efficiency and the results are given as adjusted Relative Luciferase Units (AdjRLU). Three to five independent transfections were performed in each case.

\section{Electrophoretic Mobility Shift Assay (EMSA)}

Three potential GATA sites for positions $-651,-636$ and 619 were examined by electrophoretic mobility shift assay (EMSA). The probe sequences were biotin-5-TTCCTGCCTCCATCTGGGCCCTG-3', biotin-5'-GGGCCCTGCTGATATTGTAATCA-3' and biotin-5'-TAATCA CCCTGATGCACGTTGGC-3', respectively. Nuclear extracts from HEL and MEG-01 cells were prepared as described by Andrews and Faller [52]. Three to four $\mu \mathrm{g}$ of nuclear extract were incubated with biotin-labeled probe for $20 \mathrm{~min}$ at room temperature with LightShift EMSA optimization kit reagents (Pierce, IL, USA, cat.no. 20148X) as per manufacturer's instruction. Two to four $\mu \mathrm{l}$ polyclonal anti-GATA-1 (Active Motif, Carlsbad, CA, USA), monoclonal anti-GATA-2 (Santa Cruz Biotechnology Inc., CA, USA, sc-9008) or polyclonal anti-CD63 (Santa Cruz Biotechnology Inc., CA, USA, sc-7080) antibodies were added to the reaction mixtures and incubated for $15 \mathrm{~min}$ at room temperature. A $20 \mu \mathrm{l}$ binding reaction mixture contained $1 \mathrm{x}$ binding buffer, $2.5 \%$ glycerol, $5 \mathrm{mM} \mathrm{MgCl}_{2}, 50 \mathrm{ng} / \mathrm{ul}$ poly(dI.dC), 0.05\% NP-40, and $20 \mathrm{fmol}$ biotin leveled probe. The samples were separated on a $6 \%$ DNA retardation gel (Invitrogen, UK) in $0.5 \% \mathrm{TBE}$ buffer at $90 \mathrm{~V}$ followed by semi-dry blotting to $0.45 \mathrm{~mm}$ Biodyne B pre-cut modified nylon membranes (Pierce, IL, USA) for $30 \mathrm{~min}$ at $20 \mathrm{~V}$. Immediately after blotting, DNA was cross-linked to the membrane in the GS gene linker UV chamber (Bio-Rad, CA, USA) for 55 sec $\left(120 \mathrm{~mJ} / \mathrm{cm}^{2}\right)$. The membrane was processed as per manufacturer's instruction and the chemiluminescence was determined on Hyperfilm ECL (Amersham Pharmacia, UK).

\section{Chromatin Immunoprecipitation (ChIP) assay}

ChIP was performed by use of an IP assay kit (Millipore, MA, USA). Chromatin was prepared from $10^{6} \mathrm{HEL} /$ MEG-01 cells and cross-linked with $1 \%$ formaldehyde at $37^{\circ} \mathrm{C}$ for $10 \mathrm{~min}$. Cells were washed in ice-cold PBS lacking $\mathrm{Ca}^{2+} \& \mathrm{Mg}^{2+}$ and supplemented with protease inhibitor (Roche Applied Science, IN, USA). The cell pellet was resuspended in $200 \mu \mathrm{l}$ SDS lysis buffer supplemented with protease inhibitor and incubated on ice for $10 \mathrm{~min}$. Sonication was performed on ice for 3-4 sets of $10 \mathrm{sec}$ pulses at $40 \%$ amplitude using UP 50 Ultraschallprozessor (LabVision, $\mathrm{GmbH}$ ) at an interval of $2 \mathrm{~min}$. To reduce non-specific background, sonicated samples were pre-cleared with salmon sperm DNA/protein A agarose slurry. For IP, $4 \mu \mathrm{l}$ of polyclonal anti-GATA-1 (Active Motif, Carlsbad, CA, USA) or $0.8 \mathrm{mg}$ monoclonal anti-
GATA-2 antibodies (Santa Cruz Biotechnology Inc., CA, USA, sc-9008) were added followed by rotation overnight at $4^{\circ} \mathrm{C}$. Then, $60 \mu \mathrm{l}$ salmon sperm DNA/protein A agarose slurry was added and incubated for one $\mathrm{h}$ at $4^{\circ} \mathrm{C}$ with rotation. Agarose-immunoprecipitate was collected by centrifugation and washed as per manufacturer's instruction. Histone complex was eluted with $250 \mu \mathrm{l}$ freshly prepared elution buffer (1\% SDS, 0.1M NaHCO3). Histone DNA crosslinks were reversed at $65^{\circ} \mathrm{C}$ for $4 \mathrm{~h}$ in $5 \mathrm{M} \mathrm{NaCl}$ followed by digestion with proteinase K. DNA was extracted with phenol-chloroform-isoamylalcohol. The recovered DNA was used in duplicate PCR reactions performed on each immunoprecipitated template. Forward and reverse primers for GATA sites were 5'-TCTCACACGCACCCTCTGTTTATTTTCCTGC-3' and 5'- AG AGGAGAGAAGCCAACGTGCATCAGGGTG-3'. Control forward and reverse primers were: 5'- TCTGCTCCAATATGAATATTGAACTACTTC-3' and 5'- TTGTT TTTAAATAACCCACTCACATTAACA-3'. Three different chromatin preparations were used for each IP.

\section{Quantitative real-time PCR}

Real-time PCR was performed as described previously [19]. Based on the Ct values of the samples, transcript levels were calculated from a standard curve. Relative quantification based on the $\Delta \mathrm{Ct}$ method [53] was used. Normalization: $\Delta \mathrm{Ct}=\mathrm{Ct}$ (sample) - Ct (HEL cells of corresponding dilution concentraction). Relative quantification $=2^{-\Delta C t}$. Relative mRNA level presented is relatively quantified, subtracted $\mathrm{Ct}$ value of HEL cells from the samples $\mathrm{Ct}$ value of the corresponding dilution concentration.

\section{Immunoprecipitation (IP) and Western blotting}

IP and Western blotting were performed as described previously [18]. The following antibodies were used: polyclonal anti-GATA-1 (Active Motif, Carlsbad, CA, USA), polyclonal anti-GATA-2 (R\&D Systems, MN, USA), polyclonal anti-ETO specifically reactive with ETO [54], and polyclonal anti-MTG reactive with all ETO homologues and AML1-ETO [54].

\section{Bioinformatics}

Sequences of cDNA were analyzed using the NCBI Blast program http://www.ncbi.nlm.nih.gov/BLAST/. Conserved regions were searched by multiple alignment of genomic sequences using ClustalW http:// www.ebi.ac.uk/Tools/clustalw2/index.html. Potential transcription factor binding sites were identified with MatInspector http://www.genomatix.de//matinspector.html and the Jaspar database (Jaspar.genereg.net).

\section{Statistical analysis}

The statistical significance between two samples was determined by student's t-test. 


\section{Authors' contributions}

RA carried out most of the experiments, analyzed data and was involved in drafting the manuscript. RSD initiated the project, carried out some experiments, supervised experimental design/data analysis and was involved in drafting the manuscript. UG supervised experimental design/data analysis and was involved in drafting the manuscript. IO supervised experimental design/ data analysis and was involved in drafting the manuscript. All critically revised and approved the final manuscript.

\section{Acknowledgements}

This work was supported by the Swedish Cancer Foundation and the Swedish Childhood Cancer Foundation. We are grateful to Ann-Maj Persson for kind help and expert guidance.

\section{Author Details}

'Department of Hematology, C14, BMC, S-221 84 Lund, Sweden and 2Protista Biotechnology AB, IDEON, Ole Römers väg 12, SE 22370 Lund, Sweden

Received: 5 March 2010 Accepted: 20 May 2010

Published: 20 May 2010

\section{References}

1. Feinstein PG, Kornfeld K, Hogness DS, Mann RS: Identification of homeotic target genes in Drosophila melanogaster including nervy, a proto-oncogene homologue. Genetics 1995, 140(2):573-586.

2. Chevallier N, Corcoran CM, Lennon C, Hyjek E, Chadburn A, Bardwell VJ, Licht JD, Melnick A: ETO protein of t(8;21) AML is a corepressor for Bcl-6 B-cell lymphoma oncoprotein. Blood 2004, 103(4):1454-1463.

3. Goardon N, Lambert JA, Rodriguez P, Nissaire P, Herblot S, Thibault P, Dumenil D, Strouboulis J, Romeo PH, Hoang T: ETO2 coordinates cellular proliferation and differentiation during erythropoiesis. The EMBO journal 2006, 25(2):357-366.

4. McGhee L, Bryan J, Elliott L, Grimes HL, Kazanjian A, Davis JN, Meyers S: Gfi-1 attaches to the nuclear matrix, associates with ETO (MTG8) and histone deacetylase proteins, and represses transcription using a TSAsensitive mechanism. Journal of cellular biochemistry 2003 , 89(5):1005-1018.

5. Melnick AM, Westendorf JJ, Polinger A, Carlile GW, Arai S, Ball HJ, Lutterbach B, Hiebert SW, Licht JD: The ETO protein disrupted in t(8;21)associated acute myeloid leukemia is a corepressor for the promyelocytic leukemia zinc finger protein. Molecular and cellular biology 2000, 20(6):2075-2086.

6. Schuh AH, Tipping AJ, Clark AJ, Hamlett I, Guyot B, Iborra FJ, Rodriguez P, Strouboulis J, Enver T, Vyas P, et al:: ETO-2 associates with SCL in erythroid cells and megakaryocytes and provides repressor functions in erythropoiesis. Molecular and cellular biology 2005, 25(23):10235-10250.

7. Zhang J, Kalkum M, Yamamura S, Chait BT, Roeder RG: E protein silencing by the leukemogenic AML1-ETO fusion protein. Science (New York, NY) 2004, 305(5688):1286-1289.

8. Gelmetti V, Zhang J, Fanelli M, Minucci S, Pelicci PG, Lazar MA: Aberrant recruitment of the nuclear receptor corepressor-histone deacetylase complex by the acute myeloid leukemia fusion partner ETO. Molecular and cellular biology 1998, 18(12):7185-7191.

9. Lutterbach B, Westendorf JJ, Linggi B, Patten A, Moniwa M, Davie JR, Huynh KD, Bardwell VJ, Lavinsky RM, Rosenfeld MG, et al:: ETO, a target of $\mathrm{t}(8 ; 21)$ in acute leukemia, interacts with the $\mathrm{N}-\mathrm{CoR}$ and $\mathrm{mSin} 3$ corepressors. Molecular and cellular biology 1998, 18(12):7176-7184.

10. Wang J, Hoshino T, Redner RL, Kajigaya S, Liu JM: ETO, fusion partner in $\mathrm{t}(8 ; 21)$ acute myeloid leukemia, represses transcription by interaction with the human $\mathrm{N}-\mathrm{CoR} / \mathrm{mSin} 3 / \mathrm{HDAC} 1$ complex. Proceedings of the National Academy of Sciences of the United States of America 1998, 95(18):10860-10865.

11. Hildebrand D, Tiefenbach J, Heinzel T, Grez M, Maurer AB: Multiple regions of ETO cooperate in transcriptional repression. The Journal of biological chemistry 2001, 276(13):9889-9895.

12. Amann JM, Nip J, Strom DK, Lutterbach B, Harada H, Lenny N, Downing JR, Meyers S, Hiebert SW: ETO, a target of $\mathrm{t}(8 ; 21)$ in acute leukemia, makes distinct contacts with multiple histone deacetylases and binds $\mathrm{mSin} 3 \mathrm{~A}$ through its oligomerization domain. Molecular and cellular biology 2001, 21(19):6470-6483.
13. Zhang J, Hug BA, Huang EY, Chen CW, Gelmetti V, Maccarana M, Minucc S, Pelicci PG, Lazar MA: Oligomerization of ETO is obligatory for corepressor interaction. Molecular and cellular biology 2001, 21(1):156-163.

14. Erickson P, Gao J, Chang KS, Look T, Whisenant E, Raimondi S, Lasher R, Trujillo J, Rowley J, Drabkin H: Identification of breakpoints in $\mathrm{t}(8 ; 21)$ acute myelogenous leukemia and isolation of a fusion transcript, AML1/ETO, with similarity to Drosophila segmentation gene, runt. Blood 1992, 80(7):1825-1831

15. Miyoshi H, KozU T, Shimizu K, Enomoto K, Maseki N, Kaneko Y, Kamada N, Ohki M: The t(8;21) translocation in acute myeloid leukemia results in production of an AML1-MTG8 fusion transcript. The EMBO journal 1993, 12(7):2715-2721.

16. Gamou T, Kitamura E, Hosoda F, Shimizu K, Shinohara K, Hayashi Y, Nagase $\mathrm{T}$, Yokoyama Y, Ohki M: The partner gene of AML1 in $\mathrm{t}(16 ; 21)$ myeloid malignancies is a novel member of the MTG8(ETO) family. Blood 1998, 91(11):4028-4037.

17. Wolford JK, Prochazka M: Structure and expression of the human MTG8/ ETO gene. Gene 1998, 212(1):103-109.

18. Dhanda RS, Lindberg SR, Olsson I: The human SIN3B corepressor forms a nucleolar complex with leukemia-associated ETO homologues. BMC molecular biology 2008, 9:8.

19. Lindberg SR, Olsson A, Persson AM, Olsson I: The Leukemia-associated ETO homologues are differently expressed during hematopoietic differentiation. Experimental hematology 2005, 33(2):189-198.

20. Chyla BJ, Moreno-Miralles I, Steapleton MA, Thompson MA, Bhaskara S, Engel M, Hiebert SW: Deletion of Mtg16, a target of $\mathrm{t}(16 ; 21)$, alters hematopoietic progenitor cell proliferation and lineage allocation. Molecular and cellular biology 2008, 28(20):6234-6247.

21. Calabi F, Pannell R, Pavloska G: Gene targeting reveals a crucial role for MTG8 in the gut. Molecular and cellular biology 2001, 21(16):5658-5666

22. Amann JM, Chyla BJ, Ellis TC, Martinez A, Moore AC, Franklin JL, McGhee L, Meyers S, Ohm JE, Luce KS, et al.: Mtgr 1 is a transcriptional corepressor that is required for maintenance of the secretory cell lineage in the small intestine. Molecular and cellular biology 2005, 25(21):9576-9585.

23. Tonks A, Pearn L, Tonks AJ, Pearce L, Hoy T, Phillips S, Fisher J, Downing JR, Burnett AK, Darley RL: The AML1-ETO fusion gene promotes extensive self-renewal of human primary erythroid cells. Blood 2003, 101(2):624-632

24. Choi Y, Elagib KE, Delehanty LL, Goldfarb AN: Erythroid inhibition by the leukemic fusion AML1-ETO is associated with impaired acetylation of the major erythroid transcription factor GATA-1. Cancer research 2006, 66(6):2990-2996.

25. Akashi K, Traver D, Miyamoto T, Weissman IL: A clonogenic common myeloid progenitor that gives rise to all myeloid lineages. Nature 2000, 404(6774):193-197.

26. Wasserman WW, Sandelin A: Applied bioinformatics for the identification of regulatory elements. Nature reviews 2004, 5(4):276-287.

27. Weiss MJ, Yu C, Orkin SH: Erythroid-cell-specific properties of transcription factor GATA-1 revealed by phenotypic rescue of a genetargeted cell line. Molecular and cellular biology 1997, 17(3):1642-1651.

28. Ferreira R, Ohneda K, Yamamoto M, Philipsen S: GATA1 function, a paradigm for transcription factors in hematopoiesis. Molecular and cellular biology 2005, 25(4):1215-1227.

29. Gutierrez L, Nikolic T, van Dijk TB, Hammad H, Vos N, Willart M, Grosveld F, Philipsen S, Lambrecht BN: Gata1 regulates dendritic-cell development and survival. Blood 2007, 110(6):1933-1941.

30. Ko LJ, Engel JD: DNA-binding specificities of the GATA transcription factor family. Molecular and cellular biology 1993, 13(7):4011-4022.

31. Grass JA, Boyer ME, Pal S, Wu J, Weiss MJ, Bresnick EH: GATA-1-dependent transcriptional repression of GATA-2 via disruption of positive autoregulation and domain-wide chromatin remodeling. Proceeding of the National Academy of Sciences of the United States of America 2003, 100(15):8811-8816.

32. Rylski M, Welch JJ, Chen YY, Letting DL, Diehl JA, Chodosh LA, Blobel GA, Weiss MJ: GATA-1-mediated proliferation arrest during erythroid maturation. Molecular and cellular biology 2003, 23(14):5031-5042.

33. Welch JJ, Watts JA, Vakoc CR, Yao Y, Wang H, Hardison RC, Blobel GA, Chodosh LA, Weiss MJ: Global regulation of erythroid gene expression by transcription factor GATA-1. Blood 2004, 104(10):3136-3147.

34. Oikawa T, Yamada T: Molecular biology of the Ets family of transcription factors. Gene 2003, 303:11-34. 
35. Cantor AB, Orkin SH: Transcriptional regulation of erythropoiesis: an affair involving multiple partners. Oncogene 2002, 21(21):3368-3376.

36. Kim SI, Bresnick EH: Transcriptional control of erythropoiesis: emerging mechanisms and principles. Oncogene 2007, 26(47):6777-6794.

37. Fujiwara Y, Browne CP, Cunniff K, Goff SC, Orkin SH: Arrested development of embryonic red cell precursors in mouse embryos lacking transcription factor GATA-1. Proceedings of the National Academy of Sciences of the United States of America 1996, 93(22):12355-12358.

38. Shivdasani RA, Fujiwara Y, McDevitt MA, Orkin SH: A lineage-selective knockout establishes the critical role of transcription factor GATA-1 in megakaryocyte growth and platelet development. The EMBO journal 1997, 16(13):3965-3973.

39. Vyas $P$, Ault $K$, Jackson CW, Orkin SH, Shivdasani RA: Consequences of GATA-1 deficiency in megakaryocytes and platelets. Blood 1999, 93(9):2867-2875.

40. Weiss MJ, Keller G, Orkin SH: Novel insights into erythroid development revealed through in vitro differentiation of GATA-1 embryonic stem cells. Genes \& development 1994, 8(10):1184-1197.

41. Leonard M, Brice M, Engel JD, Papayannopoulou T: Dynamics of GATA transcription factor expression during erythroid differentiation. Blood 1993, 82(4):1071-1079.

42. Martin DI, Zon LI, Mutter G, Orkin SH: Expression of an erythroid transcription factor in megakaryocytic and mast cell lineages. Nature 1990, 344(6265):444-447.

43. Shimizu R, Yamamoto M: Gene expression regulation and domain function of hematopoietic GATA factors. Seminars in cell \& developmental biology 2005, 16(1):129-136.

44. Patient RK, McGhee JD: The GATA family (vertebrates and invertebrates). Current opinion in genetics \& development 2002, 12(4):416-422.

45. Wang X, Crispino JD, Letting DL, Nakazawa M, Poncz M, Blobel GA Control of megakaryocyte-specific gene expression by GATA- 1 and FOG-1: role of Ets transcription factors. The EMBO journal 2002 . 21(19):5225-5234

46. Muntean AG, Crispino JD: Differential requirements for the activation domain and FOG-interaction surface of GATA-1 in megakaryocyte gene expression and development. Blood 2005, 106(4):1223-1231.

47. Munugalavadla V, Dore LC, Tan BL, Hong L, Vishnu M, Weiss MJ, Kapur R: Repression of c-kit and its downstream substrates by GATA-1 inhibits cell proliferation during erythroid maturation. Molecular and cellular biology 2005, 25(15):6747-6759.

48. Hamlett I, Draper J, Strouboulis J, Iborra F, Porcher C, Vyas P: Characterization of megakaryocyte GATA1-interacting proteins: the corepressor ETO2 and GATA1 interact to regulate terminal megakaryocyte maturation. Blood 2008, 112(7):2738-2749.

49. Gardini A, Cesaroni M, Luzi L, Okumura AJ, Biggs JR, Minardi SP, Venturini E, Zhang DE, Pelicci PG, Alcalay M: AML1/ETO oncoprotein is directed to AML1 binding regions and co-localizes with AML1 and HEB on its targets. PLoS genetics 2008, 4(11):e1000275

50. Lutterbach B, Sun D, Schuetz J, Hiebert SW: The MYND motif is required for repression of basal transcription from the multidrug resistance 1 promoter by the $\mathrm{t}(8 ; 21)$ fusion protein. Molecular and cellular biology 1998, 18(6):3604-3611

51. Lennartsson A, Pieters K, Ullmark T, Vidovic K, Gullberg U: AML-1, PU.1, and $\mathrm{Sp} 3$ regulate expression of human bactericidal/permeabilityincreasing protein. Biochemical and biophysical research communications 2003, 311(4):853-863.

52. Andrews NC, Faller DV: A rapid micropreparation technique for extraction of DNA-binding proteins from limiting numbers of mammalian cells. Nucleic acids research 1991, 19(9):2499.

53. Ginzinger DG: Gene quantification using real-time quantitative PCR: an emerging technology hits the mainstream. Experimental hematology 2002, 30(6):503-512.

54. Lindberg SR, Olsson A, Persson AM, Olsson I: Interactions between the leukaemia-associated ETO homologues of nuclear repressor proteins. European journal of haematology 2003, 71(6):439-447.

doi: 10.1186/1471-2199-11-38

Cite this article as: Ajore et al., The leukemia associated ETO nuclear repressor gene is regulated by the GATA-1 transcription factor in erythroid/megakaryocytic cells BMC Molecular Biology 2010, 11:38

\section{Submit your next manuscript to BioMed Central} and take full advantage of:

- Convenient online submission

- Thorough peer review

- No space constraints or color figure charges

- Immediate publication on acceptance

- Inclusion in PubMed, CAS, Scopus and Google Scholar

- Research which is freely available for redistribution

Submit your manuscript at www.biomedcentral.com/submit
C Biomed Central 